\title{
Role of biomarkers and electrocardiography assessment as individual predictors of anthracycline cardiotoxicity in lymphoma patients
}

\author{
Monika Długosz-Danecka', Andrzej Jaroszyński ${ }^{2}$, Wojciech Jurczak ${ }^{1}$ \\ 'Department of Haematology, Jagiellonian University, Cracow, Poland \\ Chair of Department: Wojciech Jurczak, prof. MD, PhD \\ ${ }^{2}$ Department of Nephrology, Family Medicine and Geriatrics, Institute of Medical Sciences, \\ Jan Kochanowski University, Kielce, Poland
}

\section{ABSTRACT}

The significant progress in the treatment of non-Hodgkin lymphomas, translating into prolongation of overall survival results in the manifestation of long-term adverse events, like anthracycline-related cardiotoxicity. Despite the dose-dependent cardiac dysfunction and the presence of risk factors, the increasing probability of cardiotoxicity arises from individual predisposition. Identification of high-risk patients gives the opportunity to implement the prevention strategies to reduce the incidence

Correspondence:

Monika Długosz-Danecka Department of Haematology, Jagiellonian University in Cracow

31-501 Cracow, Kopernika 17 phone: (+48) 606-979-167 e-mail:monika.dlugosz-danecka@uj.edu.pl ORCID number: 0000-0002-8927-4125

Received: 22.11.2018 Accepted: 27.12.2018

DOI: 10.24292/01.OR.271218 Copyright $\odot$ Medical Education. All rights reserved. of cardiac complications.

The study evaluated the utility of biomarkers: N-terminal B-type natriuretic peptide, troponin I and electrocardiography with spatial QRS-T angle assessment, as indicators of individual sensitivity of cardiomyocytes to doxorubicin resulting in myocardial damage. Thirty-five treatment-naïve patients at increased risk of cardiotoxicity, were subjected prospectively during (R)-CHOP treatment to echocardiographic assessment and analysis of biomarkers: Tnl and NT-proBNP plasma level and spatial QRS-T assessment before and 24 hours after each cycle of chemotherapy.

The analysis of QRS-T angle was consistent with the results of NT-proBNP assessment and allowed to identify, after the first cycle of chemotherapy, patients at increased risk of developing cardiovascular complications, who require thorough echocardiographic analysis and primary cardioprotection implementation. Our data did not reveal the role of $\mathrm{Tnl}$ in the identification of cardiac events. Our findings, though promising, should be confirmed in a larger group of patients in real-life or clinical trials.

Key words: biomarkers, cardiotoxicity, doxorubicin, electrocardiography, lymphoma 


\section{INTRODUCTION}

The significant progress in the treatment of non-Hodgkin lymphomas (NHL), translating into prolongation of overall survival (OS), results in the manifestation of long-term adverse reactions of applied therapies. Doxorubicin, an anti-cancer anthracycline antibiotic and a key component of CHOP (cyclophosphamide, doxorubicin, vincristine and prednisone) regimen, administered with or without rituximab $(\mathrm{R})$, the most commonly used in first-line treatment for aggressive lymphomas, is among the most potent chemotherapeutic agents which has truly revolutionized the management of $\mathrm{NHL}$ [1]. However, doxorubicin utility is compromised by cardiotoxicity, manifesting initially as asymptomatic cardiac dysfunction detected by imaging studies, heart rhythm disturbance and diastolic dysfunction, evolving irreversibly to congestive heart failure (CHF) and premature cardiac deaths [2-4]. The introduction of cardiac imaging technology and detection of even asymptomatic left ventricular (LV) dysfunction revealed the higher incidence of anthracycline-induced cardiotoxicity than previously estimated [5]. The risk for cardiac dysfunction increases with cumulative dose [6]. However, despite the established dose-dependent association and the presence of risk factors increasing the probability of cardiotoxicity $[7,8]$, there is interpatient variability in individual risk of heart failure (HF) development during lymphoma treatment $[9,10]$. Identification of high-risk patients gives the opportunity to implement the prevention strategies to reduce the incidence of cardiac complications.

\section{OBJECTIVE OF THE WORK}

The major objective of this study was to evaluate the utility of biomarkers: N-terminal B-type natriuretic peptide (NT-proBNP), troponin I (Tnl) and electrocardiography (ECG), with spatial QRS-T angle assessment, as indicators of individual sensitivity of cardiomyocytes to doxorubicin and myocardial damage.

\section{MATERIALS AND METHODS}

We evaluated 35 treatment-naïve $\mathrm{NHL}$ patients treated at the Department of Haematology of Jagiellonian University Hospital, Cracow, Poland. The diagnosis of NHL was histopathologically confirmed in all cases. Most common subtype was diffuse large B-cell lymphoma ( $n=25,71 \%$ ), followed by indolent lymphoma subtypes ( $n=3,9 \%)$, mantle cell lymphoma ( $n=5,14 \%)$ and peripheral T-cell lymphoma $(n=2,6 \%)$. All patients were at increased risk of anthracycline cardiotoxicity, defined as the existence of at least one risk factor such as: coronary artery disease, valvular heart disease, significant arrhythmia, hypertension, dia- betes, smoking, obesity, previous myocardial infarction or stroke, identified as important according to the results of multicenter Polish Lymphoma Research Group (PLRG) study [11]. Risk factors were determined at diagnosis, based on past medical history (PMH) and physical examination.

Patients were treated between 2012 and 2014 with primary cardioprotection implemented according to current standard of European Society for Medical Oncology (ESMO) [12]. All patients received 6 (R)-CHOP cycles as the first-line therapy, with $50 \mathrm{mg} / \mathrm{m}^{2}$ doxorubicin per cycle (cumulative dose of $300 \mathrm{mg} / \mathrm{m}^{2}$ ). Supportive treatment, including prevention of tumour lysis syndrome, prophylactic antibacterial, antiviral and antifungal therapy and transfusions of red blood cells, platelets or other blood products was implemented as required.

Echocardiography was performed as an element of good clinical practice, at least 3 times (at diagnosis, mid therapy and after its completion) in all patients. Laboratory tests were performed in Department of Diagnostics of University Hospital in Cracow.

Patients were subjected to the evaluation by immunochemical methods of $\mathrm{Tnl}$ and NT-proBNP in plasma samples taken prior and up to $24 \mathrm{~h}$ after anthracycline intravenous administration. All values of $\mathrm{Tnl}>0.01 \mathrm{mg} / \mathrm{l}$ and NT-proNBP $>125 \mathrm{pg} / \mathrm{ml}$ were considered as elevated. Previous studies identified the value of NT-proBNP $=400 \mathrm{pg} / \mathrm{ml}$ as cut-off for screening for patients with higher risk of left ventricular systolic dysfunction and the value of $2060 \mathrm{pg} / \mathrm{ml}$ as indicator for acute myocardial injury [13, 14]. According to these results, in our analysis patients were divided into three groups of risk, based on the levels of NT-proBNP: lower than $400 \mathrm{pg} / \mathrm{ml}$ considered to be at low risk of HF, $400-2000 \mathrm{pg} / \mathrm{ml}$ - patients at intermediate risk of HF and over 2000 pg/ml considered as patients at high risk of HF.

Resting ECG with spatial QRS-T angle assessment was performed in all patients before and 24 hours after anthracycline infusion. The 12-lead resting digital ECG was performed using the Cardiax PC. For the study, the QRS-T angle above 90 degrees was estimated as a pathological value $[15,16]$.

All patients were treated with a maximum tolerated dose of ACE-inhibitors (ramipril), except women with childbearing potential and/or beta-blockers (bisoprolol or similar), started before implementing (R)-CHOP regimen. Non-pegylated liposomal doxorubicin (NPLD) was administered to selected patients in case of contraindications to conventional doxorubicin, defined as diagnosis of heart failure before chemotherapy or occurrence 
of systolic and/or diastolic dysfunction of the left ventricle during its course.

Clinical cardiotoxicity was defined as cardiovascular death, symptoms of HF such as orthopnoea, fatigue, weakness, significantly reduced exercise tolerance, nocturnal dyspnoea, peripheral oedema or acute symptoms related to ischemic heart disease (IHD): unstable angina or myocardial infarction. Echocardiographic cardiotoxicity was identified as cardiac LV systolic dysfunction: decline in LV ejection fraction (LVEF) by $>10$ percentage points from baseline to a final value of LVEF below $50 \%$, with evaluation of impairment of LV diastolic function [17]. The grading scheme was implemented from the current echocardiographic guidelines published by the European Association of Echocardiography and the American Society of Echocardiography [18].

All statistical analyses were performed using STATISTICA software.

\section{RESULTS}

Patients were treated with a standard dose of anthracycline-containing chemotherapy: R-CHOP and CHOP (in 33 and 2 cases respectively) with any reductions of anthracycline doses. The Tnl results were not elevated neither at baseline or following the treatment (data not shown). After the analysis of NT-proBNP plasma levels before treatment and after each anthracycline administration, we identified a subset of patients with a significant rise of NT-pro BNP plasma level 24 hours after the end of the first chemotherapy cycle (fig. 1): $54 \%(\mathrm{n}=19)$ patients displayed NT-proBNP levels $>400$ and $<2000 \mathrm{pg} / \mathrm{ml}$, whereas in $9 \%(\mathrm{n}=3)$ of patients the level exceeded $2000 \mathrm{pg} / \mathrm{ml}$. After completion of treatment, NT-proBNP levels have normalized in $85.71 \%(n=30)$ of patients while in $14.29 \%$ $(n=5)$ cases remained elevated $>400$ and $<2000 \mathrm{pg} / \mathrm{ml}$. In high-risk group according to NT-proBNP plasma level all patients $(n=3)$ presented normal systolic function of LV in all analyses and $1^{\text {st }}$ grade diastolic dysfunction of LV in interim analysis, with stabilization after switching to liposomal doxorubicin. In intermediate-risk NT-proBNP patients cohort systolic dysfunction was assesed in frontline and further analysis in 1 patients, $1^{\text {st }}$ grade diastolic dysfunction of LV in interim analysis, with improvement after switching to liposomal doxorubicin was observed in 47,3\% ( $n=9$ ). In low-risk NT-proBNP patients no echocardiographic systolic or diastolic dysfunctions were found during observation (tab. 1).

All patients were also subjected to accurate ECG analysis. Systematic analysis including resting ECG with spatial QRS-T angle assessment was performed prior and $24 \mathrm{~h}$ post every chemotherapy cycle. We considered the value of the QRS-T angle $>90$ as a risk factor for cardiovascular disease (CVD). The results of the test showed QRS-T angle $>90$ in 17\% $(n=6)$ of patients. Interestingly, 3 out of 6 patients with QRS-T angle $>90$ had NT-proBNP levels > $2000 \mathrm{pg} / \mathrm{ml}$; other 3 patients displayed NT-proBNP levels $>400$ and $<2000 \mathrm{pg} / \mathrm{ml}$. Four out of 6 patients QRS-T angle $>90$ developed $1^{\text {st }}$ grade diastolic dysfunction in interim analysis

FIGURE 1.

The levels of NT-proNBP prior and post every chemotherapy cycle.

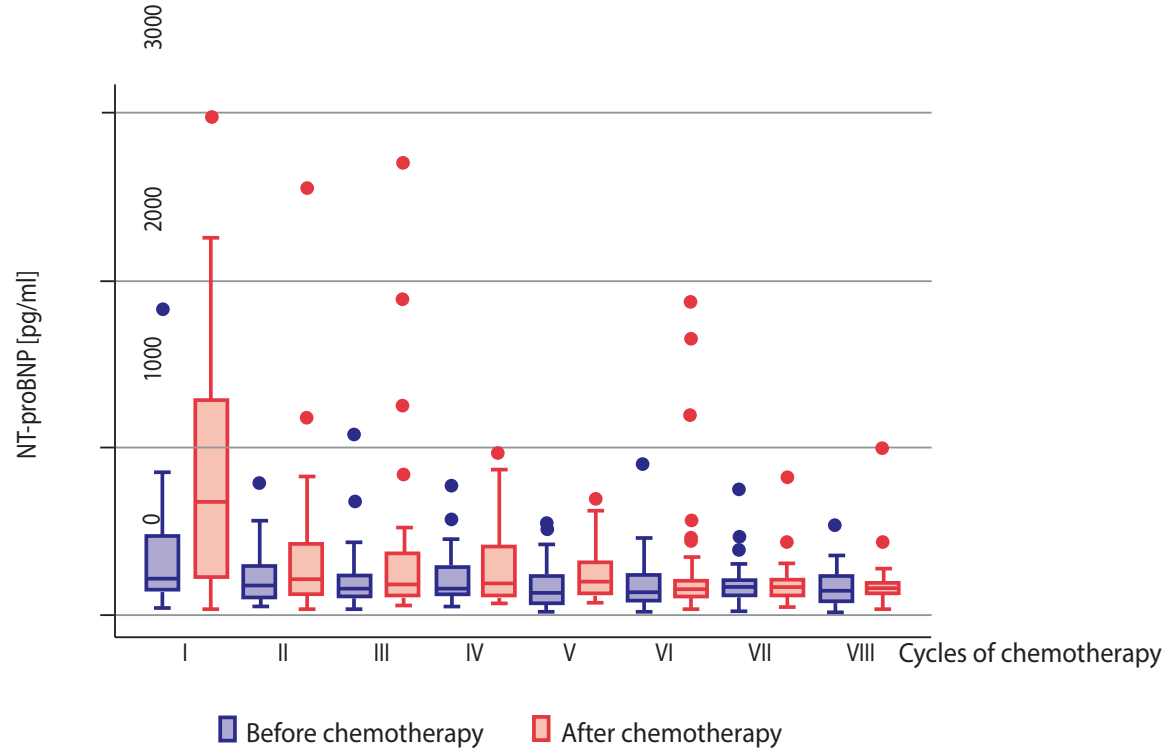


TABLE 1.

The relationship beetwen NT-proBNP levels, spatial QRS-T angle and LV systolic and diastolic disorders.

\begin{tabular}{|c|c|c|c|c|c|c|}
\hline & \multicolumn{3}{|c|}{ Systolic dysfunction } & \multicolumn{3}{|c|}{ Diastolic dysfunction } \\
\hline & $\begin{array}{c}\text { Before treatment } \\
\mathrm{n}(\%)\end{array}$ & $\begin{array}{c}\text { Interim analysis } \\
\mathrm{n}(\%)\end{array}$ & $\begin{array}{c}\text { After treatment } \\
\mathrm{n}(\%)\end{array}$ & $\begin{array}{c}\text { Before treatment } \\
\mathrm{n}(\%)\end{array}$ & $\begin{array}{c}\text { Interim analysis } \\
\mathrm{n}(\%)\end{array}$ & $\begin{array}{c}\text { After treatment } \\
\mathrm{n}(\%)\end{array}$ \\
\hline \multicolumn{7}{|c|}{ Cardiotoxicity risk according to NT-proBNP level } \\
\hline $\begin{array}{c}\text { Low } \\
(n=13)\end{array}$ & 0 & 0 & 0 & 0 & 0 & 0 \\
\hline $\begin{array}{l}\text { Intermediate } \\
\quad(n=19)\end{array}$ & $1(5.3)$ & $1(5.3)$ & $1(5.3)$ & 3 & $9(47)$ & $4(21)$ \\
\hline $\begin{array}{l}\text { High } \\
(n=3)\end{array}$ & 0 & 0 & 0 & 0 & $3(100)$ & $3(100)$ \\
\hline
\end{tabular}

with any abnormalities in systolic function of LV (tab. 1). Diastolic dysfunction withdrew after switching to liposomal doxorubicin in 3 out of 4 patients. Clinical cardiotoxicity occurred in one patient in the form of cardiogenic syncope. This patient was in high-risk group, both NT-proBNP and spatial QRS-T angle.

\section{DISCUSSION}

A key issue in the treatment of lymphoma patients is the incidence of cardiac events and cardiovascular deaths. Although extensive efforts have been devoted to identify effective primary prevention strategies and select the patients with individual susceptibility, there is a little consensus regarding the best approach. The high efficiency of cardioprotective drugs (ramipril and/or bisoprolol) in combination with NPLD can be successful in the treatment of patients with increased risk of cardiovascular complications related to anthracyclines [19]. Furthermore, there is an increasing evidence that initial damage to the heart during anthracycline-based chemotherapy is subclinical and first signs of cardiac injury may appear after first dose of doxorubicin in predisposed patients. Such an early onset may be determined by patient's individual genetic or enzymatic hypersensitivity to the drug $[20,21]$. This study placed an emphasis on finding specific cardiac biomarkers, released early during myocardial injury. Clinical trials data suggest that searching for specific markers to identify the individual risk of anthracycline cardiotoxicity may facilitate early identification of complications and identify the group of patients who benefit the most from in-depth diagnostics [22-27]. In our analysis, measurement of Tnl seemed to have no predictive value for the identification of patients at increased risk of cardiac events (data not presented). Brain natriuretic peptide (BNP) and NT-proBNP have been considered as potential biomarkers of cardiac dysfunction but their role in the management of cancer patients is much less defined compared to the general population [28]. In adult patients treated with anthracyclines, the natriuretic peptides predicted deterioration in heart function [29]. The data from analysis of leukemia patients treated with idarubicin demonstrated the significant increase of BNP plasma con- centration after the first and third dose with a trend toward the dilatation of LV [30]. Suzuki et al. also demonstrated elevated BNP levels in 27 patients with a marginal increase in E/A ratio assessed in echocardiography after anthracycline-based therapy [31]. An important finding of our analysis was that moderate or high increase in plasma NT-proBNP concentration is limited mainly to the first cycle of chemotherapy, and occurred in a subset of patients, pinpointed a group of patients with the necessity of systolic and diastolic function assessment in echocardiography. The mechanism of this phenomenon is unclear and may be caused by unidentified compensatory mechanism providing protection and limiting the damage [25]. ECG alterations may occur during chemotherapy and the most common and relevant finding consists in QT corrected (QTc) interval prolongation [32]. QTc measures the total duration of the electric depolarization and repolarization of myocardium. Its prolongation is associated to increased risk of ventricular arrhythmias, particularly torsades de pointes, and sudden death [33]. The mechanism is mediated by the interaction between chemotherapeutic agents with potassium ion channel (hERG K+). In the past, there have been many attempts to evaluate the risk of developing cardiovascular complications by analysing the QRS-T angle in resting electrocardiogram [15, 16]. The data from the analysis of 967 patients with hypertrophic cardiomyopathy (HCM) showed that the assessment of spatial QRS-T angle can differentiate patients with HCM from controls with high sensitivity and specificity [34]. Although elongation of QTc during chemotherapy is relatively frequent as presented by Naing et al. in the results of phase I clinical trial (14\% of 525 patients enrolled) [35] and ECG monitoring is recommended in the follow-up of chemotherapy-treated patients, the association between this electric disorder and major cardiac events occurrence has not yet been proved in this set of patients. Our evaluation of QRS-T angle to assess the risk of cardiotoxicity in lymphoma patients treated with (R)-CHOP regimen can allow to separate the group of patients at high risk of anthracycline induced cardiotoxicity.

The limitation of our analysis is the small sample size. 


\section{CONCLUSIONS}

The results of QRS-T angle analysis were consistent with the results of NT-proBNP assessment and allowed to identify patients with increased risk of developing cardiovascular complications, who require thorough echocardiographic analysis and primary cardioprotection implementation. Our data we did not reveal the role of Tnl in the identification of high-risk patients of cardiotoxicity. Our findings, though promising, should be confirmed in a larger group of patients in real-life or clinical trials.

\section{References}

1. Minotti G, Menna P, Salvatorelli E et al. Anthracyclines: molecular advances and pharmacologic developments in antitumor activity and cardiotoxicity. Pharmacol Rev 2004; 56(2): 185-229. DOI: 10.1124/pr.56.2.6.

2. Limat S, Demesmay K, Voillat L et al. Early cardiotoxicity of the CHOP regimen in aggressive non-Hodgkin's lymphoma. Ann Oncol 2003; 14(2): 277-281.

3. Hershman DL, McBride RB, Eisenberger A et al. Doxorubicin, cardiac risk factors, and cardiac toxicity in elderly patients with diffuse B-cell non-Hodgkin's lymphoma. J Clin Oncol 2008; 26(19): 3159-3165. DOI: 10.1200/JCO.2007.14.1242.

4. Menna P, Calabrese V, Armento G et al. Pharmacology of Cardio-Oncology: Chronotropic and Lusitropic Effects of B-Type Natriuretic Peptide in Cancer Patients with Early Diastolic Dysfunction Induced by Anthracycline or Nonanthracycline Chemotherapy. J Pharmacol Exp Ther 2018; 366(1): 158-168. DOI: 10.1124/jpet.118.249235.

5. Vejpongsa P, Yeh ET. Prevention of anthracycline-induced cardiotoxicity: challenges and opportunities. J Am Coll Cardiol 2014; 64(9): 938-945. DOI: 10.1016/j.jacc.2014.06.1167.

6. Lipshultz SE, Lipsitz SR, Sallan SE et al. Chronic progressive cardiac dysfunction years after doxorubicin therapy for childhood acute lymphoblastic leukemia. J Clin Oncol 2005; 23(12): 2629-2636. DOI: 10.1200/JCO.2005.12.121.

7. Scully RE, Lipshultz SE. Anthracycline cardiotoxicity in long-term survivors of childhood cancer. Cardiovasc Toxicol 2007; 7(2): 122-128. DOI: 10.1007/s12012-007-0006-4.

8. Armstrong GT, Oeffinger KC, Chen Y et al. Modifiable risk factors and major cardiac events among adult survivors of childhood cancer. J Clin Oncol 2013; 31(29): 3673-3680. DOI: 10.1200/JCO.2013.49.3205.

9. Armenian $\mathrm{SH}$, Ding $\mathrm{Y}$, Mills $\mathrm{G}$ et al. Genetic susceptibility to anthracycline-related congestive heart failure in survivors of haematopoietic cell transplantation. Br J Haematol 2013; 163(2): 205-213. DOI: 10.1111/bjh.12516.

10. Jensen BC, McLeod HL. Pharmacogenomics as a risk mitigation strategy for chemotherapeutic cardiotoxicity. Pharmacogenomics 2013; 14(2): 205-213. DOI: 10.2217/pgs.12.205.

11. Jurczak W, Szmit S, Sobocinski M et al. Premature cardiovascular mortality in lymphoma patients treated with (R)-CHOP regimen - a national multicenter study. Int J Cardiol 2013; 168(6): 5212-5217. DOI: 10.1016/j.ijcard.2013.08.033.

12. Curigliano G, Cardinale D, Suter T et al. Cardiovascular toxicity induced by chemotherapy, targeted agents and radiotherapy: ESMO Clinical Practice Guidelines. Ann Oncol 2012; 23(Suppl 7): vii155-66. DOI: 10.1093/annonc/mds293.

13. Davidson NC, Naas AA, Hanson JK et al. Comparison of atrial natriuretic peptide B-type natriuretic peptide, and N-terminal proatrial natriuretic peptide as indicators of left ventricular systolic dysfunction. Am J Cardiol 1996; 77(10): 828-831. DOI: 10.1016/S0002-9149(97)89176-X.

14. Gegenhuber A, Mueller T, Dieplinger B et al. B-type natriuretic peptide and amino terminal proBNP predict one-year mortality in short of breath patients independently of the baseline diagnosis of acute destabilized heart failure. Clin Chim Acta 2006; 370(1-2): 174-179. DOI: 10.1016/j. cca.2006.02.010.

15. Pavri BB, Hillis MB, Subacius $\mathrm{H}$ et al. Prognostic value and temporal behavior of the planar QRS-T angle in patients with nonischemic cardiomyopathy. Circulation 2008; 117(25): 3181-3186. DOI: 10.1161/CIRCULATIONAHA.107.733451.

16. Borleffs CJ, Scherptong RW, Man SC et al. Predicting ventricular arrhythmias in patients with ischemic heart disease: clinical application of the ECG-derived QRS-T angle. Circ Arrhythm Electrophysiol 2009; 2(5): 548-554. DOI: 10.1161/CIRCEP.109.859108.

17. Piotrowski G, Gawor R, Gawor Z et al. [Role of echocardiography in monitoring of cardiac toxicity of cancer pharmacotherapy. Expert consensus statement of the Polish Clinical Forum for Cardiovascular Imaging]. Kardiol Pol 2014; 72(6): 558-575. DOI: 10.5603/KP.2014.0126.

18. Seidman A, Hudis C, Pierri MK et al. Cardiac dysfunction in the trastuzumab clinical trials experience. J Clin Oncol 2002; 20(5): 1215-1221. DOI: 10.1200/JCO.2002.20.5.1215.

19. Dlugosz-Danecka M, Gruszka AM, Szmit S et al. Primary Cardioprotection Reduces Mortality in Lymphoma Patients with Increased Risk of Anthracycline Cardiotoxicity, Treated by R-CHOP Regimen. Chemotherapy 2018; 63(4): 238-245. DOI: 10.1159/000492942.

20. Billingham ME, Mason JW, Bristow MR, Daniels JR. Anthracycline cardiomyopathy monitored by morphologic changes. Cancer Treat Rep 1978; 62(6): 865-872.

21. Druck MN, Gulenchyn KY, Evans WK et al. Radionuclide angiography and endomyocardial biopsy in the assessment of doxorubicin cardiotoxicity. Cancer 1984; 53(8): 1667-1674.

22. Cardinale D, Sandri MT, Martinoni A et al. Myocardial injury revealed by plasma troponin I in breast cancer treated with high-dose chemotherapy. Ann Oncol 2002; 13(5): 710-715.

23. Cardinale D, Sandri MT, Colombo A et al. Prognostic value of troponin I in cardiac risk stratification of cancer patients undergoing high-dose chemotherapy. Circulation 2004; 109(22): 2749-2754. DOI: 10.1161/01.CIR.0000130926.51766.CC.

24. Dolci A, Dominici R, Cardinale D et al. Biochemical markers for prediction of chemotherapy-induced cardiotoxicity: systematic review of the literature and recommendations for use. Am J Clin Pathol 2008; 130(5): 688-695. DOI: 10.1309/AJCPB66LRIIVMQDR.

25. Ekstein S, Nir A, Rein AJ et al. N-terminal-proB-type natriuretic peptide as a marker for acute anthracycline cardiotoxicity in children. J Pediatr Hematol Oncol 2007; 29(7): 440-444. DOI: 10.1097/MPH.0b013e3180640d42. 
26. Horacek JM, Vasatova M, Pudil R et al. Biomarkers for the early detection of anthracycline-induced cardiotoxicity: current status. Biomed Pap Med Fac Univ Palacky Olomouc Czech Repub 2014; 158(4): 511-517. DOI: 10.5507/bp.2014.004.

27. Sandri MT, Salvatici M, Cardinale D et al. N-terminal pro-B-type natriuretic peptide after high-dose chemotherapy: a marker predictive of cardiac dysfunction? Clin Chem 2005; 51(8): 1405-1410. DOI: 10.1373/clinchem.2005.050153.

28. Zamorano JL, Lancellotti P, Rodriguez Munoz D et al. 2016 ESC Position Paper on cancer treatments and cardiovascular toxicity developed under the auspices of the ESC Committee for Practice Guidelines: The Task Force for cancer treatments and cardiovascular toxicity of the European Society of Cardiology (ESC). Eur J Heart Fail 2017; 19(1): 9-42. DOI: 10.1002/ejhf.654.

29. Okumura $\mathrm{H}$, luchi $\mathrm{K}$, Yoshida T et al. Brain natriuretic peptide is a predictor of anthracycline-induced cardiotoxicity. Acta Haematol 2000; 104(4): 158-163. DOI: 10.1159/000046508.

30. Nousiainen T, Jantunen E, Vanninen E et al. Acute neurohumoral and cardiovascular effects of idarubicin in leukemia patients. Eur J Haematol 1998; 61(5): 347-353.

31. Suzuki T, Hayashi D, Yamazaki T et al. Elevated B-type natriuretic peptide levels after anthracycline administration. Am Heart J 1998; 136(2): 362-363. DOI: 10.1053/hj.1998.v136.89908.

32. Bagnes C, Panchuk PN, Recondo G. Antineoplastic chemotherapy induced QTc prolongation. Curr Drug Saf 2010; 5(1): 93-96.

33. Trinkley KE, Page RL $2^{\text {nd }}$, Lien H et al. QT interval prolongation and the risk of torsades de pointes: essentials for clinicians. Curr Med Res Opin 2013; 29(12): 1719-1726. DOI: 10.1185/03007995.2013.840568.

34. Cortez D, Schlegel TT, Ackerman MJ, Bos JM. ECG-derived spatial QRS-T angle is strongly associated with hypertrophic cardiomyopathy. J Electrocardiol 2017; 50(2): 195-202. DOI: 10.1016/j.jelectrocard.2016.10.001.

35. Naing A, Veasey-Rodrigues H, Hong DS et al. Electrocardiograms (ECGs) in phase I anticancer drug development: the MD Anderson Cancer Center experience with 8518 ECGs. Ann Oncol 2012; 23(11): 2960-2963. DOI: 10.1093/annonc/mds130.

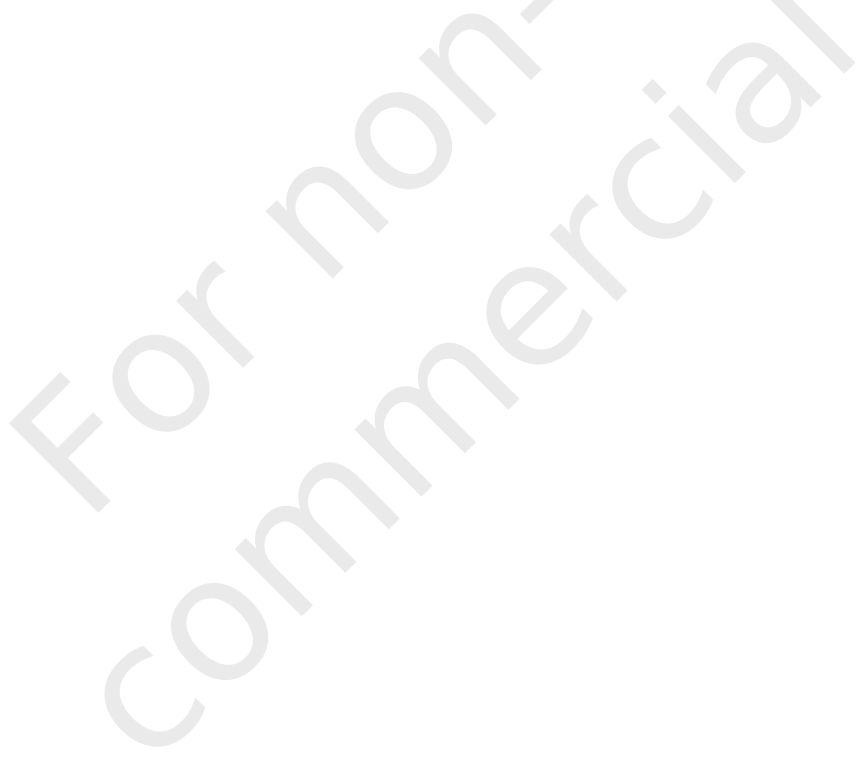

Authors' contributions:

Monika Długosz-Danecka: performed the study and analysed the data, had substantial contributions to the conception and design of the work, drafted the manuscript, critically revised the manuscript for important intellectual content, had substantial contributions in the acquisition, analysis and interpretation of data for the work

Wojciech Jurczak: designed the study, had substantial contributions to the conception and design of the work, critically revised the manuscript for important intellectual content, had substantial contributions in the acquisition, analysis and interpretation of data for the work Andrzej Jaroszyński: designed the study, had substantial contributions to the conception and design of the work, critically revised the manuscript for important intellectual content, had substantial contributions in the acquisition, analysis and interpretation of data for the work.

Ethics:

The authors had full access to data and take full responsibility for their integrity. All authors have read and agreed with the content of the manuscript as written. All authors declare no conflict of interest. The work described has been carried out in accordance with The Code of Ethics of the World Medical Association (Declaration of Helsinki) and EU2010/63/EU Directive for experiments involving humans. Informed consent was obtained for experimentation with human subjects. This article does not contain any studies with animals performed by any of the authors. 